Original Article

\title{
Diagnostic value of urinary interleukin-6 in urinary tract infection in children.
}

\author{
Huda Marzouk $^{1}$, Emad E. Ghobrial ${ }^{1}$, Mervat M Khorshied ${ }^{2}$, Safaa G Samuel ${ }^{3}$ \\ 1- Department of Pediatrics, Faculty of Medicine, Cairo University, Cairo. \\ 2- Department of Clinical Pathology, Faculty of Medicine, Cairo University, Cairo. \\ 3- Department of Pediatrics, Ministry of Health, Cairo.
}

\begin{abstract}
Introduction

Urinary tract infection (UTI) is the second most common bacterial infection in children.
\end{abstract}

\section{Aim of the study}

To estimate the value of urinary Interleukin-6 (IL-6) in diagnosis of UTI in children and also if it can differentiate between upper and lower UTI.

\section{Patients and Methods}

This study included 80 children, divided into 40 patients suffering from UTI and 40 healthy children served as a control group. Patients were classified into acute pyelonephritis $(n=22)$ and lower UTI $(n=18)$. Urine IL-6 was measured by enzyme-linked immunesorbent assay (ELISA) technique.

\section{Results}

Urinary IL-6 was significantly higher in patients with UTI than control group ( $p$ value $<0.001)$ and also it was significantly higher in upper than lower UTI cases ( $\mathrm{p}$ value $<0.001$ ).

\section{Conclusion}

Urinary IL-6 level is a reliable diagnostic tool for early identification of UTI in febrile children. The study confirms usefulness of urinary IL-6 to differentiate between upper and lower UTIs.

\section{Keywords}

UTI; upper and lower UTI; urinary interleukin-6

\section{Running title}

Urinary tract infection, IL-6.

\section{Corresponding Author Emad E. Ghobrial}

Department of Pediatrics, Faculty of Medicine, Cairo University, Cairo.

Mailing address $72^{\text {nd }}$ Mohamed Abdel-Moneam Street, off Rayel Street- Helwan-Cairo-Egypt.

E-mail dr.emademil@yahoo.com

Reprints addressed to Emad E. Ghobrial

Mobile: 0201222383671

\section{geget: The Journal of the Egyptian Society of Pediatric Nephrology and Transplantation (ESPNT)}

$$
\begin{aligned}
& \text { geget https://geget.journals.ekb.eg/ } \\
& \text { Published by ESPNT http://espnt.net/ }
\end{aligned}
$$

\section{Cohosted by Egyptian Knowledge Bank https://www.ekb.eg}




\section{Introduction}

Urinary tract infections (UTIs) represent the second most common bacterial infection in children. It may be localized to the kidney (upper UTI) and/or bladder (lower UTI) or spread to the tissue outside the urinary tract. In the majority of cases, it responds adequately to treatment $[1,2]$. Distinction between cystitis and pyelonephritis is problematic. Clinical findings (such as fever or flank pain) and inflammatory markers such as, erythrocyte sedimentation rate [ESR], C-reactive protein [CRP], and total leukocyte count (TLC) are suggestive and not conclusive in distinguishing pyelonephritis from cystitis [3].

Acute pyelonephritis (APN) is one of the most common serious bacterial infections when it affect infants and young children. The most significant complication of acute pyelonephritis (APN) is permanent renal damage, characterized by proteinuria, hypertension and chronic kidney disease [4]. Predicting which children with upper UTI will develop longterm sequel remains difficult [2].

Differentiating acute pyelonephritis from lower UTIs is important because the accurate diagnosis and prompt treatment of APN can prevent renal scarring and subsequent complications [5].Although determination of inflammatory markers, such as CRP and ESR are recommended for the evaluation of children with APN [6] yet their sensitivity and specificity are not that high .Fever is not a strong indicator of the presence of acute inflammatory lesions in the kidneys of younger infants and children. Inflammatory markers including $\mathrm{C}$-reactive protein (CRP), white blood cells count (WBC) and erythrocyte sedimentation rate (ESR) are also unreliable diagnostic indicators in the acute phase of pyelonephritis[3].Procalcitonin is accepted as the most reliable marker that localize upper UTI. In Acute pyelonephritis, bacteria stimulate renal epithelial cells to produce a number of cytokines and chemokines, among other substances [7].Cytokines, especially interleukin-6 (IL-6), participate in the local inflammatory response to infections. Therefore Elevated levels of urinary IL-6 and IL- 8 have been observed in patients with UTI [8].

Interleukin-6 (IL-6) is a pro-inflammatory cytokine that is synthesized by various cells, including macrophages, fibroblasts, endothelial cells, and renal tubular epithelial cells. It acts as a pyrogen appearing early during the inflammatory process, and it is responsible for the acute-phase reaction, including the development of fever and the increased production of acute phase proteins, such as $\mathrm{C}$ - reactive protein. It is also a key inducer of $\mathrm{B}$ and $\mathrm{T}$-cell activation and differentiation during inflammation [9].

The aim of the study was to evaluate the efficacy of urinary IL-6 in diagnosis of acute urinary tract infection in children and its value in differentiation between upper and lower UTI.

\section{Patients and Methods}

The present study was carried out during the period between June2015 to June 2016. Forty pediatric patients suffering from urinary tract infection were selected randomly from pediatric nephrology clinic and inpatient department at Children Hospital of our university.
This study included 40 pediatric patients suffering urinary tract infection suggested by clinical symptoms and confirmed by at least one positive urine culture. In addition to the patient group, 40 apparently healthy age- and sex- matched children served as a control group (21 males and 19 females). Informed consent for participation was obtained from all parents of the participants.

Patient group included infants and children 6 months to 12 years, of both sexes (22 males and 18 females) with documented UTI. Children with known concomitant diseases (allergies, rheumatological diseases or neoplasms), those who were diagnosed as had immunological or inflammatory disorders and those who have signs of any other infection requiring antibiotic treatment were excluded from the study.

The patients were divided into two groups according to level of infection (lower urinary tract infection and pyelonephritis) depending on history (high fever, lumbar pain and other constitutional symptoms like vomiting in cases of pyelonephritis and dysuria, frequency, incontinence, suprapupic pain in lower UTI), examination (temperature $>38.5$, tender renal angle, some of them had bad general condition in pyelonephritis and temperature $>38$, suprapupic mass or tenderness in lower UTI), laboratory investigations (high TLC, CRP and ESR in cases of pyelonephritis) and imaging (renal ultrasound and renal scan).

Patients were subjected to the following

I- Detailed history taking; including duration of illness, number of attacks, and presence of hypertension, drug therapy if present, fever, chills, flank pain, dysuria, frequency and vomiting.

II- Complete clinical examination; including temperature, presence of hypertension, flank tenderness, abdominal distension and suprapubic tenderness.

III- Laboratory investigations:

1. Complete blood picture (CBC): It was done using coulter auto-analyzer Pentra-80 automated blood cell counter.

2. C-reactive protein (CRP): It was done by latex agglutination test. When serum containing greater than 0.6 $\mathrm{mg} / \mathrm{dl} \mathrm{CRP}$ is mixed with the latex reagent, visible agglutination occurs within two minutes.

3. Renal function testing: Biochemical tests for renal function (blood urea nitrogen and creatinine) were done on synchron CX5 auto-analyzer using kit supplied by Beckman.

4. Urinalysis: Mid-stream urine samples were obtained from the studied subjects in plastic sterile containers after instructing them to clean the genital area with soap and tap water. Urine samples were examined either immediately or, if not possible, refrigerated at $4{ }^{\circ} \mathrm{C}$ to be examined within 24 hours.

a) Macroscopic examination: Urine was examined by the naked eye for turbidity or frank hematuria.

b) Microscopic examination: A drop of uncentrifuged urine was placed on a slide with a coverslip and examined directly without staining by X10, X40 for the presence of pus cells.

5. Urine culture: Culture of the urine samples was done on appropriate media (MacConkey agar or CLED agar plates) and incubated at $37^{\circ} \mathrm{C}$ for $24 \mathrm{~h}$ and for additional 
$24 \mathrm{~h}$ if no growth appeared. After incubation of the plates at $37^{\circ} \mathrm{C}$ for $24-48 \mathrm{~h}$ in aerobic environment, any bacterial growth obtained was further identified by examination of their colony morphology, gram stained films, and biochemical reactions. The number of colonies appearing after incubation was multiplied by 100 to detect the number of bacteria/ml urine. 6 . Urinary interleukin-6 (uIL-6) assay:

Human urine IL-6 was measured by enzyme-linked immunosorbent assay (ELISA) using Orgenium

Laboratories' Interleukin-6 (IL-6).

7. Imaging:

a) Renal ultrasonogram performed within 48 hours did not modify management.

b) Voiding cystourethrogram (VCUG) was done after 2 weeks (after complete recovery from infection).

c) Renal scan (DMSA Scan) was performed after subsidence of the attack for all cases.

\section{Statistical analysis}

All data were collected on standardized forms, entered in a computerized database, and analyzed with statistical software packing SPSS version 17.Results were statistically presented in terms of (Range, mean, median, standard deviation and percentage). Continuous data (of both groups) were compared with paired T-tests and categorical data (parametrical data) by Pearson's chi-square test was performed. A p-value $<0.05$ was considered statistically significant.

\section{Results}

Table (1) shows comparison between cases and controls as regards age and sex. There were no statistically significant differences between the 2 groups.

Escherichia coli was isolated from the urine of 24 patients $(60 \%)$, Pseudomonas aurginosa from the urine of 6 patients $(15 \%)$, Klebsiella pneumonia from the urine of 4 patients $(10 \%)$, Proteus from the urine of 2 patients $(5 \%)$, Actinobacter from the urine of 1 patient $(2.5 \%)$, Staphylococcus aureus from the urine of 1 patient $(2.5 \%)$, Streptreptococcus fecalis from the urine of 1 patient $(2.5 \%)$ and Citrobacter from the urine of 1 patient $(2.5 \%)$.Organisms isolated from urine culture of patients were highly sensitive to aminoglycoside in 10 patients $(25 \%)$, cotrimoxazole in 10 patients $(25 \%)$, imipenem in 6 patients $(15 \%)$, ampicillin-sulbactamin 3 patients (7.5\%), amoxicillinclavulinic acid in 3 patients $(7.5 \%)$, ceftriaxone in 4 patients $(10 \%)$ and azithromycin in 4 Patients $(10 \%)$.

Figure (1) shows comparison between patients and controls as regards urinary interleukin-6 (uIL-6). The mean value of urinary
Interleukin-6 (IL-6) in group I was $21.065 \pm 3.287$, while in group II the mean value of IL-6 was $7.710 \pm 4.028$. There was a highly statistically significant difference in its mean levels among the two groups ( $\mathrm{p}$ value $<0.001$ ).

Table (2) shows comparison between lower urinary tract infection and pyelonephritis regarding vital signs. There were highly statistically significant differences between lower UTI and pyelonephritis cases as regards temperature and blood pressure ( $\mathrm{p}$ value $<0.001$ and 0.031 respectively). There were only $13.64 \%$ less than $5^{\text {th }}$ percentile in weight and $4.55 \%$ less than $5^{\text {th }}$ centile in height of patients with pyelonephritis.

Table (3) shows comparison between lower urinary tract infection and pyelonephritis regarding GFR, creatinine level and inflammatory markers. It revealed highly statistically significant differences as regards GFR and serum creatinine level ( $P$ value $<0.002$ and 0.023 , respectively) and as regards TLC, CRP and uIL-6 (P value $<0.001$, each).

Table (4) shows comparison between lower urinary tract infection and pyelonephritis regarding imaging (ultrasonography, VCUG and renal scan). It revealed highly statistically significant differences between them as regards the renal ultrasonogram ( $\mathrm{P}$ value $<0.002)$, VCUG $(\mathrm{P}$ value $<0.001)$ and renal scan (P value $<0.001)$.

Table (5) shows correlations of urinary IL-6 level with renal US, VCUG and renal isotopic scan. Table (6) shows correlation between urinary IL-6 and age, duration of illness, temperature, GFR and laboratory investigations. It shows a highly statistically significant positive correlations between urinary IL-6 and temperature $[\mathrm{r}=0.521, \mathrm{P}<0.001]$, total leucocytic count (TLC) $[\mathrm{r}=+0.410, \mathrm{P}<0.009]$ and $\mathrm{C}$-reactive protein $(\mathrm{CRP})[\mathrm{r}=+0.437$, $\mathrm{P}<0.005]$.

Figure (2) shows receiver operating characteristic (ROC) graph analysis for urinary IL-6, which was applied in order to quantities and assess its diagnostic performance. It revealed that the best cutoff value of urinary IL- 6 was $>15.5 \mathrm{pg} / \mathrm{ml}$ as the diagnostic sensitivity was $97.56 \%$, specificity was $97.44 \%$, positive predictive value was $97.6 \%$, negative predictive value was $97.4 \%$ and diagnostic accuracy was $97.9 \%$. Figure (3) shows ROC curve between lower urinary tract infection and pyelonephritis cases. It revealed that the best cut-off value of urinary IL-6 for differentiation between upper and lower UTI was $>22.1 \mathrm{pg} / \mathrm{ml}$ as the diagnostic sensitivity was $50 \%$, specificity was $94.44 \%$, positive predictive value was $91.7 \%$ negative predictive value was $60.7 \%$ and diagnostic accuracy was $76 \%$.

Table 1 Descriptive statistics of the studied groups ( 80 children)

\begin{tabular}{|c|c|c|c|c|}
\hline & \multicolumn{2}{|c|}{ Gro ups } & \multirow{2}{*}{ P-value } \\
\hline & & Patients $(n=40)$ & Controls $(n=40)$ & \\
\hline \multirow{2}{*}{ Age (Years) } & Range & $0.5-12$ & $0.5-10$ & \multirow{2}{*}{0.615} \\
\hline & Mean \pm SD & $4.138 \pm 3.389$ & $3.794 \pm 2.657$ & \\
\hline \multirow{2}{*}{ Sex } & Male & $22(55 \%)$ & $21(52.5 \%)$ & \multirow{2}{*}{0.823} \\
\hline & Female & $18(45 \%)$ & $19(47.5 \%)$ & \\
\hline
\end{tabular}


Table 2 Comparison between lower urinary tract infection and pyelonephritis regarding vital signs

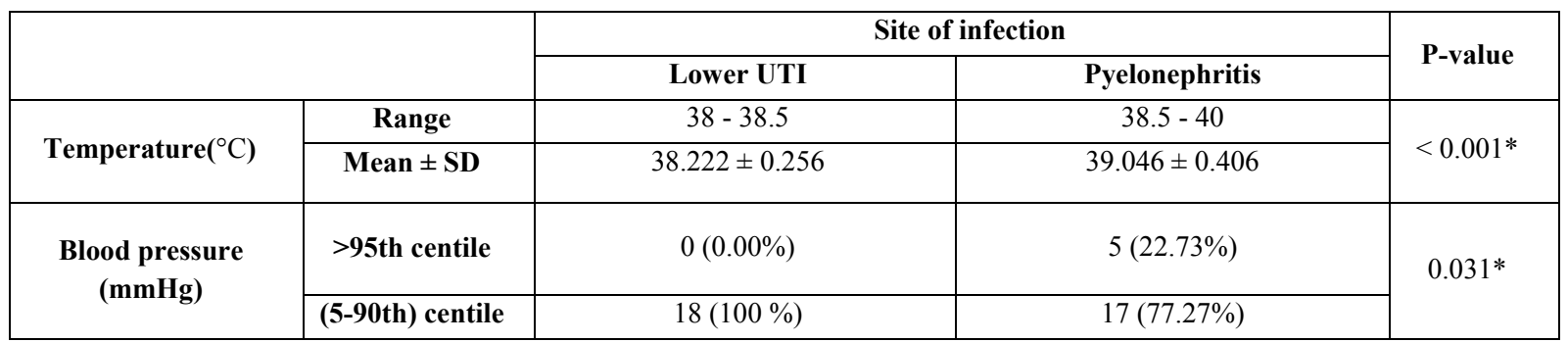

UTI: Urinary tract infection

Table 3 Comparison between lower urinary tract infection and pyelonephritis regarding GFR, creatinine level and inflammatory markers

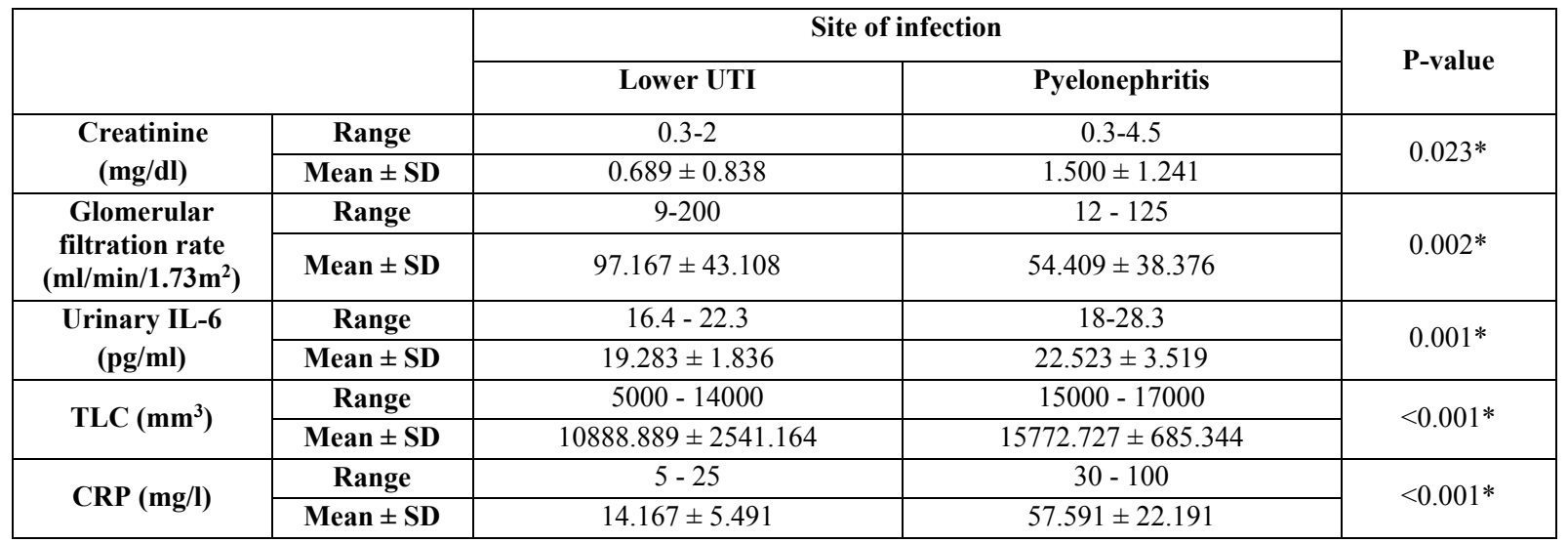

TLC: Total leukocytic count

IL-6: Interleukin-6

CRP: C-reactive protein

UTI: Urinary tract infection

Table 4 Comparison between lower urinary tract infection and pyelonephritis regarding imaging

\begin{tabular}{|c|c|c|c|c|c|c|c|c|}
\hline & & \multicolumn{6}{|c|}{ Site of infection } & \multirow{3}{*}{ P-value } \\
\hline & & \multicolumn{2}{|c|}{ Lower UTI } & \multicolumn{2}{|c|}{ Pyelonephritis } & \multicolumn{2}{|r|}{ Total } & \\
\hline & & $\mathbf{N}$ & $\%$ & $\mathbf{N}$ & $\%$ & $\mathbf{N}$ & $\%$ & \\
\hline \multirow[t]{3}{*}{ Renal US } & $\begin{array}{c}\text { Unilateral hydroureter and } \\
\text { hydronephrosis }\end{array}$ & 3 & 16.67 & 5 & 22.73 & 8 & 20.00 & \multirow{3}{*}{$0.002 *$} \\
\hline & $\begin{array}{c}\text { Bilateral hydroureter and } \\
\text { hydronephrosis }\end{array}$ & 7 & 38.89 & 17 & 77.27 & 24 & 60.00 & \\
\hline & Normal renal US & 8 & 44.44 & 0 & 0.00 & 8 & 20.00 & \\
\hline \multirow{4}{*}{ VCUG } & Unilateral reflux grade $(1+2)$ & 2 & 11.11 & 0 & 0.00 & 2 & 5.00 & \multirow{4}{*}{$0.001 *$} \\
\hline & $\begin{array}{c}\text { Bilateral reflux high grade } \\
(4+5)\end{array}$ & 1 & 5.56 & 10 & 45.45 & 11 & 27.50 & \\
\hline & $\begin{array}{l}\text { Bilateral reflux (low grade } \\
\qquad(1+2+3)\end{array}$ & 2 & 11.11 & 8 & 36.36 & 10 & 25.00 & \\
\hline & Normal VCUG & 13 & 72.22 & 4 & 18.18 & 17 & 42.50 & \\
\hline \multirow{2}{*}{ Renal scan } & No scar & 18 & 100.00 & 0 & 0.00 & 18 & 45.00 & \multirow{2}{*}{$<0.001^{*}$} \\
\hline & Scar formation & 0 & 0.00 & 22 & 100.00 & 22 & 55.00 & \\
\hline
\end{tabular}

VCUG: Voiding cystourethrogram US: Ultrasound UTI: Urinary tract infection 
Table 5 Correlation of urinary IL-6 with renal US, VCUG and renal isotopic scan

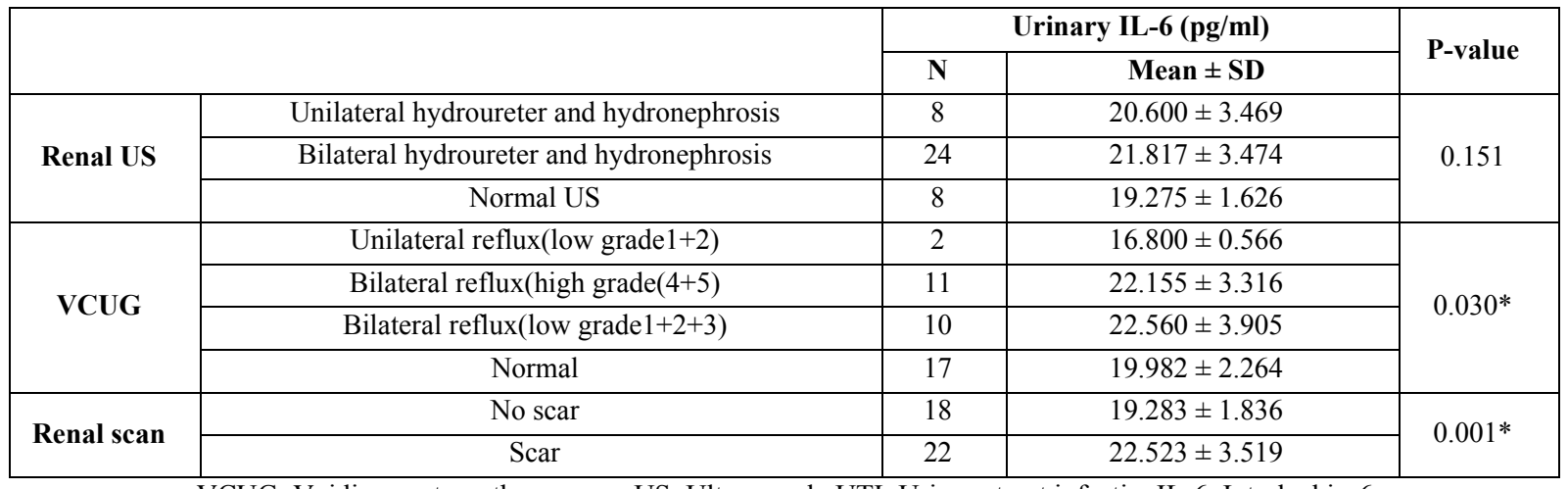

VCUG: Voiding cystourethrogram US: Ultrasound UTI: Urinary tract infectionIL-6: Interleukin-6

Table 6 Correlation between urinary IL-6 and age, duration of illness, temperature and their laboratory measurements among cases

\begin{tabular}{|c|c|c|}
\hline \multicolumn{2}{|c|}{ Correlations } & \multicolumn{2}{|c|}{ Urinary IL6 (pg/ml) } \\
\hline & P-value \\
\cline { 2 - 3 } & -0.041 & 0.802 \\
\hline Age(Years) & -0.102 & 0.533 \\
\hline Duration of illness(Years) & 0.521 & $0.001^{*}$ \\
\hline Temperature $\left({ }^{\circ} \mathbf{C}\right)$ & -0.140 & 0.389 \\
\hline GFR & 0.044 & 0.786 \\
\hline Creatinine $(\mathbf{m g} / \mathbf{d l})$ & 0.410 & $0.009^{*}$ \\
\hline TLC $(\mathbf{c m m})$ & 0.437 & $0.005^{*}$ \\
\hline
\end{tabular}

TLC: Total leukocytic count IL-6: Interleukin-6 CRP: C-reactive protein UTI: Urinary tract infection GFR: Glomerular filtration rate

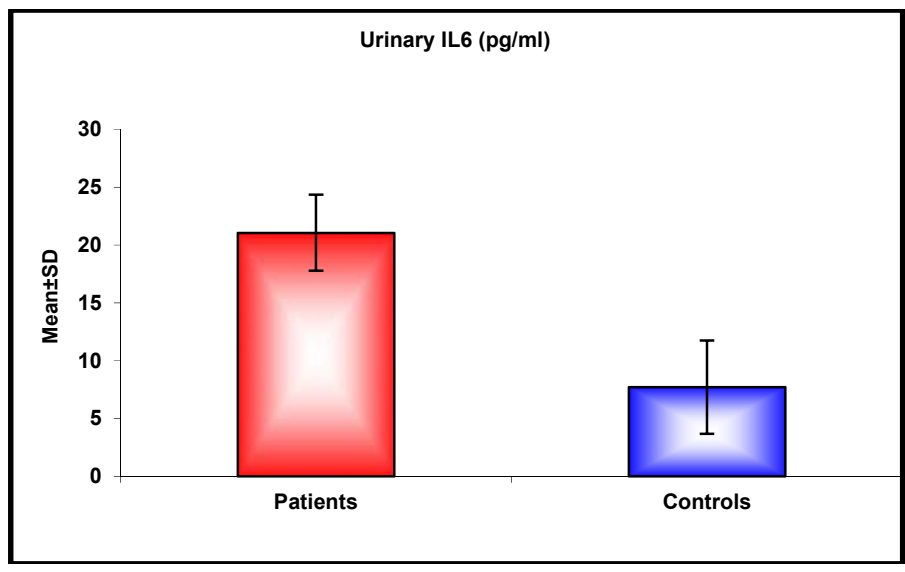

Figure 1 Comparison between patients and controls as regards urinary IL-6.

IL-6: Interleukin-6 


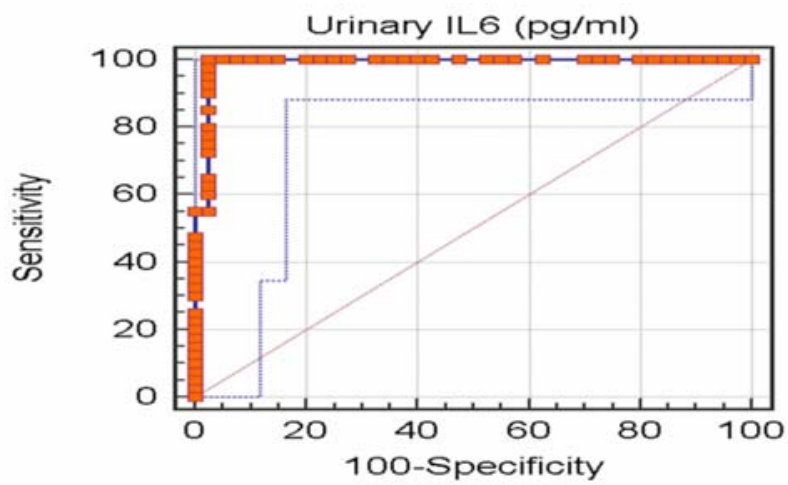

Figure 2 ROC curve for uIL-6

ROC: receiver operating characteristicuIL-6: Urinary interleukin-6

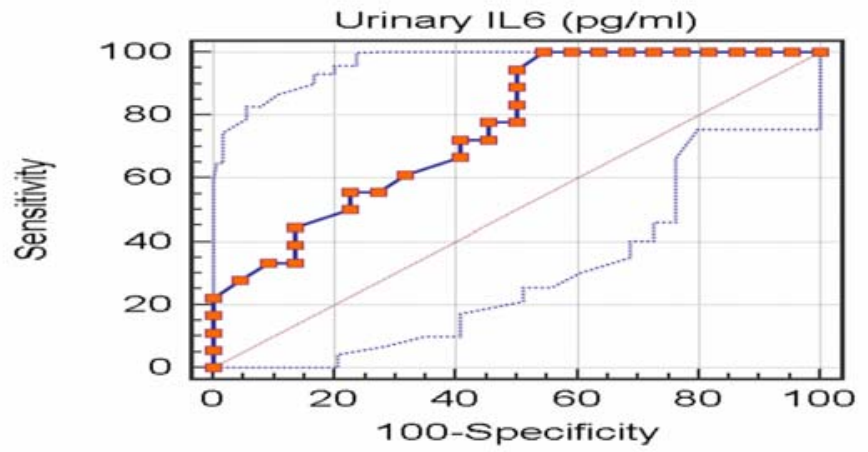

Figure 3 ROC curve between lower urinary tract infection and pyelonephritis cases.

ROC: receiver operating characteristicuIL-6: Urinary interleukin-6

\section{Discussion}

In clinical practice, acute pyelonephritis (APN) and lower UTIs are two common forms of UTI that occur during infancy and childhood. Lower UTIs do not cause serious complications, while APN may result in renal scarring and subsequent complications, such as hypertension and chronic kidney disease [10].

The diagnosis of urinary tract infection (UTI) in children is based on significant bacteriuria. The severity of infection is of great importance for the choice of treatment and prognosis. Because the clinical symptoms are nonspecific, the diagnosis of renal infection needs to be supported by laboratory data and radiologic imaging [11].

Serum and urinary levels of IL-6 protein increase during UTI, and its measurement has been shown to be useful in differentiating between APN and lower UTI. Measurement of urinary IL-6 is less aggressive than determination of serum levels and therefore more acceptable to patients and health professionals [12].

In the present study, urine culture results obtained from urine of the patients revealed infection by E. Coli in $(60 \%)$ of patient. This is in agreement with many other studies conducted on UTI in children such as Eldin et al., [13]who documented that E. coli was the most common uropathogen, accounting for $79 \%$ of urinary isolates overall.

In this study, blood pressure (BP) was significantly higher in pyelonephritis than lower UTI, in agreement with Elder et al.,[14] who found that acute pyelonephritis is the most severe type of the disease and that delay in diagnosis and treatment may lead to renal scarring, hypertension and chronic kidney disease. In our study, it was found that the mean level of inflammatory markers namely TLC and CRP were significantly higher in acute pyelonephritis than lower UTI (P value $<0,001$ ).

A study done by Gurgoze et al., [15] on 76 children with UTI showed that inflammatory markers such as CRP, ESR, WBC count, procalcitonin, IL-6, and IL- $1 \beta$ in patients with acute pyelonephritis were significantly higher than lower UTI. In our study, we found that urine levels of IL-6 were significantly increased in all patients with UTI compared to the control normal group as the diagnostic sensitivity was $97.56 \%$, specificity was $97.44 \%$, so urinary interleukin- 6 level $>15.5 \mathrm{pg} / \mathrm{ml}$ is the best cut off value for early recognition of acute urinary tract infection. Also our study proved that urinary IL-6 levels increased significantly more in acute pyelonephritis cases than in lower UTI, with sensitivity $94.44 \%$ and specificity $50 \%$ at a cut off value $>22.1$. 
This is in agreement with Rodríguez et al.,[16]who showed that, based on specificity and sensitivity of the measurement for urinary interleukin 6 (uIL-6), in the presence of clinical manifestations indicative of UTI, a concentration $>15 \mathrm{pg} / \mathrm{ml}$ is a highly reliable marker of APN. Also proved that urinary interleukin- 6 was useful in distinguishing between upper and lower urinary tract infections in children.

Sheu et al., [12] found that urine level of IL-6 was significantly increased in children with acute pyelonephritis than in lower UTI. They found also both serum and urine levels of IL6 have high sensitivity $(81-88 \%)$ and adequate specificity (78$83 \%$ ) for the diagnosis of children with acute pyelonephritis and allow accurate differentiation from lower UTI. Also Gurgoze et al., [15] found that serum IL-6 levels rose significantly in children with acute pyelonephritis, and it had $88 \%$ sensitivity and $74 \%$ specificity. On the contrary, a study done by Mahyar et al., [17] showed that sensitivity and specificity of IL-6 and IL-8 are less than those of acute phase reactants such as CRP and evaluated that these cytokines are not reliable markers for differentiating acute pyelonephritis from lower UTI.

In our study, serum creatinine levels were significantly higher in the patients with pyelonephritis compared to the cases with lower urinary tract infection. Also GFR was more deteriorated in pyelonephritis than lower UTI. This is in agreement with Fidan et al., [18] who found that cases with pyelonephritis has been associated with glomerular hyperfiltration, proteinuria, reninmediated hypertension, and deterioration of GFR in children.

In our study, there were highly statistically significant positive correlation between urinary IL- 6 and TLC, CRP and temperature, while there was no statistically significant correlation between urinary IL-6 and creatinine. This is in agreement with Sheu et al., [12] who found that in children with acute pyelonephritis, urine IL-6 was significantly correlated with fever and CRP, but does not correlate with WBC.

In our study, we found that there was highly statistically significant correlations between pyelonephritis cases and ultrasonography (US), voiding cystourethrogram (VCUG) and renal scan. There were highly statistically significant correlations between urinary IL- 6 and VCUG ( $p$ value $<0.030$ ), renal scan ( $p$ value $<0.001)$, but there was no statistically significant correlation between urinary IL- 6 and renal US ( $p$ value $<0.151$ ). Similarly, a study done by Gokce et al., [9] found that a positive correlation between IL-6 levels and the VUR reflux grade. Also, Sheu et al., [12] found that all the patients with acute pyelonephritis had positive findings in renal ultrasound. A study done by Mahyar et al., [17] found that abnormal renal ultrasound was observed in 54\% with acute pyelonephritis cases and $10 \%$ with cystitis which was statistically significant. A major limitation of this study was the small sample size and lack of follow up.

\section{Conclusion}

Our study concluded that urine IL-6 level may serve as a reliable diagnostic tool for the early identification of acute urinary tract infection in febrile children (diagnostic accuracy is $97.9 \%$ ). Also our study confirmed the usefulness of determining urinary IL-6 to differentiate between upper and lower UTIs in children. In spite of being an expensive test, yet its benefits may exceed its cost as it make the use of more aggressive diagnostic tests unnecessary.

Further studies, involving more patients are needed to study urinary IL-6 for further diagnostic accuracy for pyelonephritis and its use as an early marker in cases with suspected pyelonephritis.

\section{References}

1. Quigley R. Diagnosis of urinary tract infections in children. Curr Opin Pediatr. 2009; 21: 194-8.

2. Renata Y, Jassar H, Katz R, Hochberg A, Nir R and Klein - Kremer A.Urinary concentration of cytokines in children with acute pyelonephritis. Eur J Pediatr. 2013; 172: 76974 .

3. Ilyas M, Mastin ST and Richard GA. Age-related radiological imaging in children with acute pyelonephritis. Pediatr Nephrol. 2002; 17: 30-4.

4. Nickavar A and Sotoudeh K. Treatment and prophylaxis in pediatric urinary tract infection. Int J Prev Med. 2011; 2: 49.

5. Parenti GC and Passari A. Acute pyelonephritis. Role of diagnostic imaging. Radiol Med. 2001; 101: 251-4

6. Nickavar A, Safaeian B and Biglari M.Radiologic and clinical evaluation of children with first febrile urinary tract infection. International Journal of Pediatrics and Adolescent Medicine. 2015; 2: 24-8.

7. Hertting O, Khalil A, Jaremko G, Chromek M, Li YH, Bakhite M, Bartfai T, Tullus K and Brauner A. Enhanced chemokine response in experimental acute Escherichia coli pyelonephritis in IL-1beta-deficient mice. Clin Exp Immunol. 2003; 131: 225-33.

8. Tramma D, Hatzistylianou M, Gerasimou G and Lafazanis V. Interleukin- 6 and interleukin- 8 levels in the urine of children with renal scarring. Pediatr Nephrol. 2012; 27: 1525-30.

9. Gokce I, Alpay H, Biyikli N, Unluguzel G, Dede F and Topuzoglu A.Urinary levels of interleukin-6 and interleukin-8 in patients with vesicoureteral reflux and renal parenchymal scar. Pediatr Nephrol. 2010; 25:90512 .

10. Ghasemi K, Montazeri S, Pashazadeh AM, Javadi H and Assadi M .Correlation of 99mTc-DMSA scan with radiological and laboratory examinations in childhood acute pyelonephritis: a time-series study. Int Urol Nephrol. 2013; 45: 925-32.

11. Ataei N, Madani A, Habibi R andKhorasani M. Evaluation of acute pyelonephritis with DMSA scans in children presenting after the age of 5 years. Pediatr Nephrol. 2005; 20: 1439-44.

12. Sheu J, Chen M, Lue K, Cheng SL, Lee IC, Chen SM and Tsay GJ.Serum and urine levels of interleukin-6 and interleukin-8 in children with acute pyelonephritis Cytokine. 2006; 36: 276-82.

13. Eldin RS, Shapiro DJ, Hersh AL and Copp HL .Antibiotic resistance patterns of outpatient pediatric urinary tract infections .J Urol. 2013; 190: 222-7. 
14. Elder JS. Urinary tract infection. In: Kliegman RM, Stanton BF, St. Geme JW, Schor NF, Behrman RE, editors, Nelson textbook of pediatrics. 19th ed. Philadelphia: Saunders, 2011:1829-38.

15. Gürgôze MK, Akarsu S, Yilmaz E, Gôdekmerdan A, Akça Z, Cftçi I and Aygun AD. Proinflammatory cytokines and procalcitonin in children with acute pyelonephritis. Pediatr Nephrol. 2005; 20: 1445-8.

16. Rodríguez LM, Robles B, Marugán JM, Suárez A and Santos F.Urinary interleukin-6 is useful in distinguishing between upper and lower urinary tract infections. Pediatr Nephrol. 2008; 23: 429-33.

17. Mahyar A, Ayazi P, Maleki MR, Daneshi-Kohan MM, Sarokhani HR, Hashemi HJ and TalebiBakhshayesh M. Serum levels of interleukin- 6 and interleukin-8 as diagnostic markers of acute pyelonephritis in children. Korean J Pediatr. 2013; 56: 218-23.

18. Fidan K, Kandur Y, Buyukkaragoz B, Akdemir UO and Soylemezoglu O.Hypertension in pediatric patients with renal scarring in association with vesicoureteral reflux. Urology. 2013; 81: 173-7.

\section{Statements}

Ethics approval and consent to participate

This study protocol and consents were approved and deemed sufficient by the ethical scientific committee of the Cairo University hospital and was conducted in accordance with the University bylaws for human research. It conforms to the provisions of the Declaration of Helsinki in 2000. Informed written consent was obtained in every case from their legal guardians.

\section{Consent for publication}

Not applicable.

Availability of data and materials

Not applicable.

Conflict of interest

The authors declare no conflict of interest relevant to this article. Funding

The authors declare that this research work did not revise any fund.

Acknowledgments

Authors thank all the patients who have participated in the study and their parents. 